\title{
Inspiratory muscle training for reducing postoperative pulmonary complications: A simple method that we hope is effective
}

\author{
Chien-Hong Chou, MD, and Chung-Yu Chen, MD, PhD
}

\footnotetext{
From the Division of Pulmonary and Critical Care Medicine, Department of Internal Medicine, National Taiwan University Hospital Yunlin Branch, Yunlin County, Taiwan, and College of Medicine, National Taiwan University, Taipei, Taiwan.

Disclosures: Authors have nothing to disclose with regard to commercial support.

Received for publication March 6, 2018; accepted for publication March 9, 2018; available ahead of print April 5, 2018.

Address for reprints: Chung-Yu Chen, MD, PhD, Division of Pulmonary and Critical Care Medicine, Department of Internal Medicine, National Taiwan University Hospital Yunlin Branch, No. 579, Section 2, Yunlin Rd, Douliu City, Yunlin County, Taiwan (E-mail: c8101147@ms16.hinet.net).

J Thorac Cardiovasc Surg 2018;156:1301-2

$0022-5223 / \$ 36.00$

Copyright (C) 2018 by The American Association for Thoracic Surgery

https://doi.org/10.1016/j.jtcvs.2018.03.031
}

In their article in this issue of the Journal, "Inspiratory Muscle Training Is Associated With Decreased Postoperative Pulmonary Complications: Evidence From Randomized Trials," Ge and colleagues ${ }^{1}$ attempt to determine whether preoperative inspiratory muscle training (IMT) is associated with a significant difference in the rate of postoperative pulmonary adverse outcomes in patients undergoing cardiothoracic or upper abdominal surgery. They use the statistical method of "trial sequential analysis" to complete the meta-analysis and conclude that preoperative IMT is associated with improved maximum inspiratory pressure and decreased postoperative pulmonary complications (PPCs).

There are multiple risk factors predicting the development of PPCs, and some are modifiable. ${ }^{2}$ In many clinical trials, the use of preoperative IMT has seemed to decrease the incidence of PPCs. Most trials, however, have not been doubleblind. Previous meta-analyses ${ }^{3-5}$ have also indicated the effectiveness of IMT, but the quality of evidence is low or moderate. In the study of Ge and colleagues, ${ }^{1}$ the IMT effect is little different from that seen in previous meta-analyses. Only 1 additional recent clinical trial has been added to the current literature. The current study of Ge and colleagues ${ }^{1}$ thus faces the same problem as other meta-analyses: the evidence level is questionable.

Another limitation of the study is the incomplete documentation of some pretreatment variables (such as preoperative pulmonary test results) and of the ratio of laparoscopic or thoracoscopic surgery to open surgery. This lack of complete data is a difficulty inherent in meta-analyses. Getting information from original clinical trials is a hallmark of metaanalysis, but sometimes it is not so easy. Minimally invasive surgery may wash out the effect of IMT. Although the current meta-analysis of $\mathrm{Ge}$ and colleagues ${ }^{1}$ and the previous metaanalyses favor IMT, the evidence strength is not great enough before excluding pretreatment and operation variables. to more patients.

\section{References}

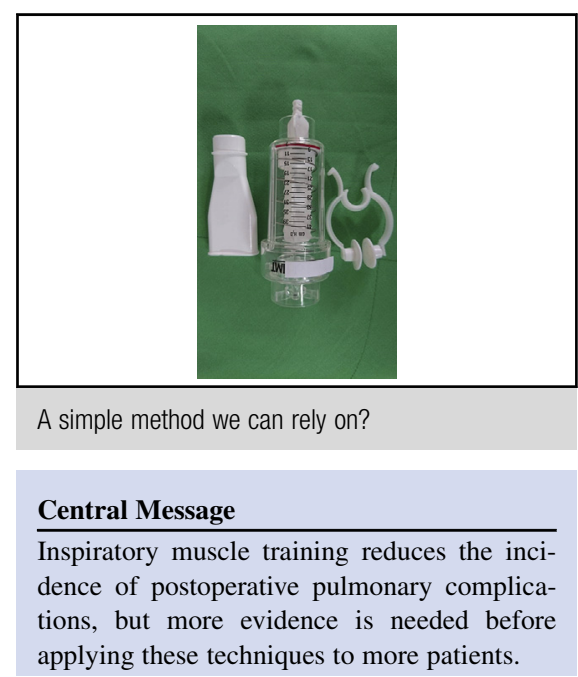

See Article page 1290.

According to previous clinical studies, most trials include IMT sessions that last more than 15 minutes a day for training periods longer than 2 weeks. This intervention is time-consuming and usually requires a physiotherapist. It is difficult to justify the application of preoperative IMT to every patient undergoing cardiothoracic or upper abdomen surgery if the evidence level is not high. IMT is therefore preferred for patients with a high risk of PPCs. Other interventions, such as preoperative smoking cessation, correcting preoperative severe anemia, avoidance of general anesthesia in favor of regional anesthesia, and protective ventilation, also have an effect on PPC prevention. ${ }^{6}$ Compared with these strategies, which are not time-consuming, IMT should have more supportive clinical evidence before being applied

In conclusion, the current study of Ge and colleagues ${ }^{1}$ strengthens the case for IMT's effect in preventing PPCs demonstrated in previous trials. More double-blind studies are needed, however, to meet a higher evidence level justifying application of this technique to more patients undergoing cardiothoracic or upper abdomen surgery.

1. Ge X, Wang W, Hou L, Yang K, Fa X. Inspiratory muscle training is associated with decreased postoperative pulmonary complications: evidence from randomized trials. J Thorac Cardiovasc Surg. 2018;156:1290-300. 
2. Smetana GW, Lawrence VA, Cornell JE, American College of Physicians. Preoperative pulmonary risk stratification for noncardiothoracic surgery: systematic review for the American College of Physicians. Ann Intern Med. 2006;144:581-95.

3. Katsura M, Kuriyama A, Takeshima T, Fukuhara S, Furukawa TA. Preoperative inspiratory muscle training for postoperative pulmonary complications in adults undergoing cardiac and major abdominal surgery. Cochrane Database Syst Rev. 2015;CD010356.
4. Mans CM, Reeve JC, Elkins MR. Postoperative outcomes following preoperative inspiratory muscle training in patients undergoing cardiothoracic or upper abdominal surgery: a systematic review and meta analysis. Clin Rehabil. 2015;29:426-38.

5. Gomes Neto M, Martinez BP, Reis HF, Carvalho VO. Pre- and postoperative inspiratory muscle training in patients undergoing cardiac surgery: systematic review and meta-analysis. Clin Rehabil. 2017;31:454-64.

6. Miskovic A, Lumb AB. Postoperative pulmonary complications. Br J Anaesth. 2017;118:317-34. 